\title{
Exploring the Flipped Learning Approach within a Mathematics Higher Education Milieu in the Era of the Fourth Industrial Revolution
}

\author{
Jayaluxmi Naidoo \\ Mathematics and Computer Science Education Cluster, School of Education, College of Humanities, University of KwaZulu-Natal, \\ South Africa
}

Received March 24, 2020; Revised April 27, 2020; Accepted May 20, 2020

Copyright $@ 2020$ by authors, all rights reserved. Authors agree that this article remains permanently open access under the terms of the Creative Commons Attribution License 4.0 International License

\begin{abstract}
We have entered the era of the Fourth Industrial Revolution, to be successful within this era, as teacher educators, we need to transform teaching and learning. We need to use pedagogy suitable for the Fourth Industrial Revolution. This paper draws attention to the future of mathematics teacher education by showcasing a flipped learning setting. This qualitative, interpretive study was framed using Activity Theory and was located at one university in KwaZulu-Natal, South Africa. Mathematics education students were observed while learning within a flipped learning setting. Subsequently, a sample of participants were interviewed using a semi-structured interview schedule. Thematic coding and interpretive techniques were used to analyze the data generated. Four main themes emerged. These themes are: The flipped learning approach is inhibited by the lack of material resources, the flipped learning approach is dependent on lecturer professional development, the flipped learning approach saves time in the lecture room and the flipped learning approach encourages interaction and collaboration within the mathematics lecture room. Globally, these findings have relevance when considering the role of technology-based tools within mathematics Higher Education lecture rooms in the era of the Fourth Industrial Revolution.
\end{abstract}

Keywords Activity Theory, Collaboration, Flipped Learning, Fourth Industrial Revolution, Mathematics, Technology-based Tools

\section{Introduction}

Education plays an important part in transforming the world in which we live. Since we have entered the era of the Fourth Industrial Revolution, technology is frequently used in contemporary society [1] and has led to transformations in our daily lives. Moreover, digital, audio and visual tools, are gradually replacing traditional pedagogy at educational institutions. Research suggests that the use of Web based technology, the internet and the use of Information and Communication Technology (ICT) could bring about a transformed educational milieu. Using ICT within education settings has become the catalyst of change [2] and consequently, within mathematics lecture rooms the use of technology-based pedagogy is popular. Through the use of technology-based pedagogy students are provided with education contexts that are relevant to their lives thereby enriching learning and encouraging success [3]. This paper focuses on: Exploring the flipped learning approach within a mathematics Higher Education lecture room in the era of the Fourth Industrial Revolution. This paper reports on a study which sought to explore mathematics students' perceptions on the use of the flipped learning approach within mathematics education. The paper responds to the question: What are students' perceptions on the use of the flipped learning approach within their mathematics lecture room? The paper is organized into six sections, namely, introduction, literature review, theoretical considerations, materials and methods, results and discussion and lastly the conclusion.

\subsection{Literature review}

\subsubsection{Fourth Industrial Revolution}

The Fourth Industrial Revolution (4IR) involves innovative competencies for people and machines and represents novel methods in which technology becomes embedded within society [4]. Within the 4IR, robotics, the 
Internet of Things (IoT), virtual reality (VR) and artificial intelligence (AI) are transforming the way we live and work. Thus, as we embark on the Fourth Industrial Revolution, it is evident that technology will play a central role in nearly all aspects of our lives. However, the rapid pace of this transformation is influencing industry and education in every country. The 4IR has transformed the way we live, work, and relates to each another [4] and this era is characterized by the merging of the physical and the virtual world, creating a more globally connected innovative society. Within traditional lecture rooms students are situated at desks surrounding one another, however within the contemporary lecture room, we envisage a transformation and that real-time collaboration will be possible globally through the use of the internet and technology-based tools. As professionals within the education sector, we need to embrace this transformation. We need to understand that what our jobs are today might be diverse in the future. Our education and training systems ought to be modified to better prepare students for the flexibility and critical thinking skills they will need in the future workplace [5].

To prepare the capacity needed for the digital economy, education must adapt as fast as the demand for Information Communications and Technology (ICT) skills is growing and evolving. The 4IR influences the roles that Higher Education institutions play in preparing students for this technologically advanced society. Within the 4IR, it is crucial to enhance the student's ability to problem-solve. Based on critical and creative thinking, problem-solving is important for flexibility and this is essential for succeeding within the 4IR. Thus, when teaching mathematics, educators need to ensure that students are motivated to solve problems and in this way students are expected to think about real world situations. To take advantage of 4IR opportunities, we need to transform our education, we need to adopt an interdisciplinary approach to Science, Technology, Engineering, and Mathematics (STEM) subjects. Mathematics, in particular, has been identified as a way of opposing unemployment and stimulating continued socio-economic existence.

\subsubsection{Exploring the use of technology-based tools within the mathematics lecture room}

Rasanen et al. [6] maintains that the use of technology-based tools in education is increasing as the technology-based tools become more popular and affordable. In addition, Pope and Mayorga [7] claimed that there are a plethora of websites and an increasing number of educational applications that may have an impact on students' learning and achievement within mathematics. In keeping with these notions, many education settings are moving forward to include more mobile and contemporary digital technologies such as tablet computers, laptops, net books, social media and collaborative platforms such as Google Drive/Classroom or Wikispaces.
Research [8] has indicated that the use of technology-based tools when teaching mathematics has enhanced student performance in mathematics. Additionally, the use of technology-based tools in mathematics classrooms may support both students that are struggling as well as those students that are successful [9]. However, for the lecturer to select the appropriate technology-based tool to navigate the vast assortment of resources is a daunting task. The idea is to select the most appropriate technology-based tool, which will allow the lecturer to exploit the full potential of the available technology to advance students' learning. It is up to the lecturer to decide which technology-based tool would be most appropriate. Thus, it is the lecturer's role to facilitate innovation in education [10], the lecturer ought to try innovative pedagogy with the aim of achieving success in the lecture room.

Teaching is evolving just as rapidly as the industries they serve, so we need to equip our lecturers with the latest innovative teaching methods. These methods will assist our lecturers to prepare future leaders. Moreover, since we are moving from teacher-centered instruction to learner-centered learning [11], one advanced method to consider is the Flipped Learning approach. The Flipped Learning approach is an innovative method that may be used in mathematics education, whereby the traditional teaching method is reversed or flipped [12].

\subsubsection{Flipped Learning Approach}

The flipped learning approach refers to an approach that is a blend between the traditional teaching approach and teaching via technology-based tools [13]. This approach requires a change in how traditional teaching and learning occurs since traditional teaching and learning transforms within the flipped learning setting. It is founded on the notion of exchanging in class teaching time with out of class practice time [11]. In order to engage with and incorporate the flipped learning approach within a lecture room, lecturers ought to integrate the four pillars of flipped learning within their lecture preparation. The four pillars are Flexible Environment, Learning Culture, Intentional Content and Professional Educator (F.L.I.P) [14].

The flexible environment requires that the lecturer construct flexible settings where students are provided with the opportunity to select when and where they will learn [15]. This was evident in this study when students were provided with the opportunity to choose to learn the uploaded content at home or during free time at the university. Within a flipped learning context, in-class time is allocated for engaging with content that was viewed prior to the lecture in order to generate meaningful learning opportunities [14]. Within the lecture room under focus, the learning culture encouraged the students to actively engage and collaborate with the lecturer and peers in order to advance their knowledge of the concepts under discussion. This interaction and collaboration also assisted 
the student to assess their own understanding. In addition, lecturers promoting the flipped learning model establish what they need to teach and what materials students need to work with independently. Content is intentional within this environment so as to optimize lecture time to promote effective and meaningful learning [16]. The role of a professional educator is also important and challenging within the flipped lecture room. This implies that during lecture time, lecturers ought to carefully observe students and offer prompt feedback and assessment of students' work [14].

Thus, by using the flipped learning approach, students view content in the form of videos and presentations prior to the lesson, hence, the use of technology is of utmost importance within the flipped learning environment. Students and the lecturer engage actively within the lecture room based on preparations done prior to the lecture. This type of learning approach requires commitment from both the student and the lecturer. Within this learning environment, the lecturer is a guide or a facilitator. Within the mathematics lecture room, there are various technology-based tools to assist both the student and the lecturer. Tools, resources and websites for example Geogebra, Geometer's Sketchpad, Mathway, Mathwords, Khan Academy provide assistance for both the student and lecturer [12]. The flipped learning method is being considered by lecturers as an advanced and effective pedagogical strategy [11].

Essentially, the flipped learning approach requires the lecturer to transform direct learning within a large lecture room to an individual learning space through the use of technology-based tools [17]. Within the flipped learning environment, the student engages with key content prior to the lecture and during the lecture/teaching time the content is discussed with peers and the facilitator so as to expand understanding. Moreover, problem-solving activities are conducted based on content being discussed. So essentially prior to attending the lecture, students engage with content in order to prepare to participate in the upcoming lecture and during the lecture students discuss their understanding with each other and through this discussion they extend and expand on their knowledge. During these discussions, the lecturer facilitates and guides the student. The lecturer provides key information via videos and presentations prior to the lecture and during the lecture, the lecturer supports students to actively discuss, solve problems and construct knowledge [11].

\subsection{Theoretical Considerations}

Activity theory is a framework that researchers use to plan and examine exchanges between members of a group with the aim of determining which aspects of these exchanges prompt online collaborations [18]. The different aspects that were considered within this study were the link between the teaching and learning of mathematics, students and the flipped learning approach. In this research study, the student was regarded as the subject, the object was the understanding of mathematics concepts and processes, the tool was the use of a flipped learning approach and the outcome was the successful teaching and learning of mathematics.

Essentially the theoretical framework was used as a structure to explore how the flipped learning approach was used as a tool to support students during the teaching and learning of mathematics. Based on the principles of activity theory, activity and learning are interactive and interdependent [19]. This theory is based on the assumption that all human actions are mediated by tools and cannot be detached from the social milieu within which actions are carried out. This theory allowed for the consideration of the relationship between the student (subject), understanding mathematics concepts and processes (object) and the use of the flipped learning approach (tool or artefact) in order to obtain the successful teaching and learning of mathematics (outcome).

The activity system under focus is depicted in Figure 1 that follows. 


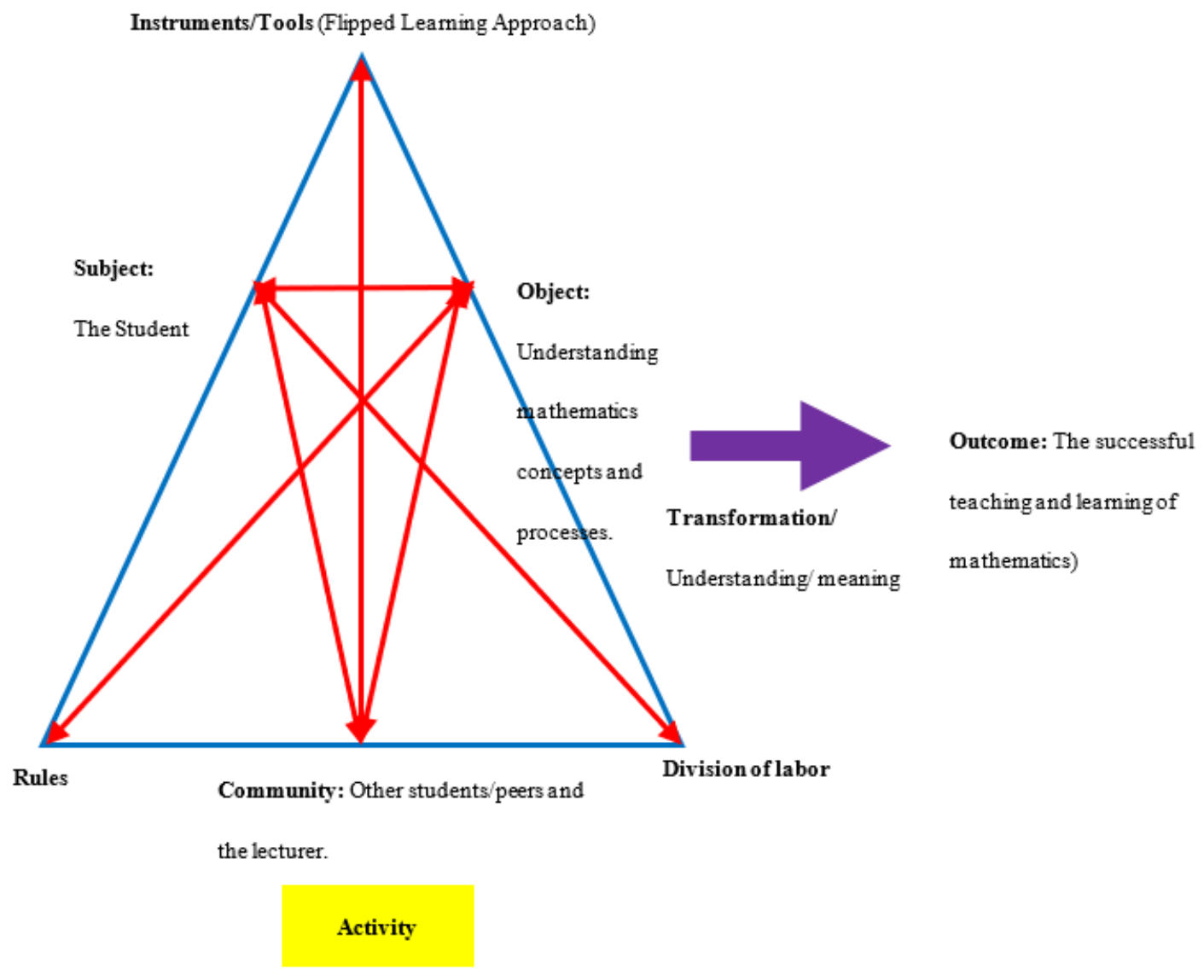

Figure 1. Based on Engeström’s [20] conceptualization. This model indicates the second generation activity system and represents the activity system under focus

In order to achieve the outcome of successful teaching and learning of mathematics, it was necessary to construct certain objects which included for example knowledge, experiences and understanding of key mathematics concepts and processes. Additionally, the student (subject) shared responsibility with the community (other students/peers and the lecturer) for the achievement of the outcome. This was recognized through a division of labor, whereby the lecturer and students responded with respect to the roles they played and the responsibilities they shared. For example on the most basic level, the student was responsible for viewing a video and completing a mathematics question based on the video prior to attending the lecture, and if there was an issue with understanding, then the student was responsible for asking for help during the discussion session in the lecture room, it was then the responsibility of the other students/peers or the lecturer to assist with clarifying, expanding on the question or supporting the student through a step by step process of solving the mathematics question during the lecture time.

Thus, it was evident that mediation through tools and technology does not occur in isolation, but rather human activity is social and collective and is achieved with the help of a community and the assistance of tools. The community in this context refers to the lecturer and other students within the lecture room. Moreover, the tool (flipped learning approach) will have an influence over the interaction between the subject (student) and the object (what is made available to the student to promote the learning and understanding of mathematics concepts and processes), hence, in this study, the technology-based tools being used is not regarded as the object of learning but rather it is perceived as the mediator of learning whereby the flipped learning approach through the use of technology-based tools is used as a tool to support students' learning $[21,22]$. There were also the rules about the knowledge, experiences and understanding that was acceptable, access to tools and artefact and who was permitted to complete aspects of the activity that were considered within this activity system. For example, the lecturer would provide feedback regarding the mathematical processes that were considered appropriate while the students would discuss possible alternative versions of solving the same question. In addition, the rule that participants don't reprimand, humiliate or cause harm to each other was also agreed upon before teaching and learning within the flipped learning environment commenced.

Thus, it was evident from this study that collaborative learning using technology-based tools within a flipped 
learning environment was situated within activities [22]. Therefore, the activities within this study were not arbitrary, but rather, they were focused to achieve a desired outcome, which was the successful teaching and learning of mathematics. The activities within this study were mediated via technology-based tools, whereby the division of labor was represented by assigning tasks which required collaborative engagement and validation of solutions using a student-centered approach. Hence, the division of labor within an activity system plays a significant role in the development of a common goal [23]. Similarly, through the use of technology-based tools, the subject (student) used the tool (the flipped learning approach) to achieve the outcome (the successful teaching and learning of mathematics) of the activity system under focus. The use of the flipped learning approach in this study supported a more successful pedagogy within a digital environment and activity theory in this study offered a possible framework for describing the structure, development and context for the activities that were supported through the use of the flipped learning approach as a tool for learning mathematics.

\section{Materials and Methods}

This qualitative study sought to explore mathematics students' perceptions on the use of the flipped learning approach within mathematics education. This study which was situated within a mathematics Higher Education lecture room was located within an interpretive paradigm. Data were generated via observations of lectures and semi-structured interviews. Gatekeeper access was obtained from the research office of the University within which this study was located. Participants were provided with an informed consent form describing the process of the study.

\subsection{The Participants}

The population for the study were postgraduate mathematics education students. These students were also mathematics school teachers. Forty five participants were invited to participate in the study and 32 responded positively. Ten participants were randomly selected to participate in the pilot study. Twenty two participants participated in the main study. Data were collected through observations of lectures and semi-structured interview schedules. Although 22 participants attended the lectures, due to personal, family, work or study obligations only 13 participants were available to be interviewed. To assure participants of their anonymity, pseudonyms were used. Table 1 that follows depicts the participants who participated in the interviews.

Table 1. Participants who participated in the semi-structured interviews

\begin{tabular}{|c|c|c|c|}
\hline Participant \# & Pseudonym & Participant \# & Pseudonym \\
\hline 1 & Jane & 8 & Sam \\
\hline 2 & Brian & 9 & Alec \\
\hline 3 & Veena & 10 & Simon \\
\hline 4 & Nomsa & 11 & Emma \\
\hline 5 & Kate & 12 & Vernon \\
\hline 6 & Busi & 13 & Bongani \\
\hline 7 & Rani & & \\
\hline
\end{tabular}

\subsection{The Pilot Study}

Conducting the pilot study lectures and semi-structured interviews improved the validity and reliability of the research process. During the pilot study, one video clip was problematic so this needed to be replaced and some participants were unclear of what was required of them for certain questions during the interview process. To eliminate ambiguity and to ensure that each interview question was comprehensible, questions were rephrased. The language used during the lecture and interview process was focused and well-defined to improve the trustworthiness and validity of the research instruments and process.

\subsection{The Lectures}

A series of lectures $(\mathrm{N}=5)$ focusing on the flipped learning approach was conducted with the participants. The lectures were facilitated by the researcher and the participants were provided with teaching notes, presentations of case studies, lesson plans and assessments focusing on the effective integration of technology-based tools within a flipped mathematics lecture room. The first three lectures focused on teaching measurement (area, perimeter and volume) to junior secondary school learners (Grade 7-9) 1 . For these three lectures, the technology-based tools integrated within the lecture included PowerPoint presentations, videos and the document camera 2. Lectures four and five focused on teaching Euclidean Geometry to senior secondary school learners (Grade 10-12) 3. For these two lectures, the technology-based tools integrated within the lecture included PowerPoint presentations, a dynamic mathematics software program (Geometer's Sketchpad) 4

1 Secondary school education in South Africa consists of 2 bands or phases: The General Education and Training (GET) and the Further Education and Training (FET) band/phase. The senior phase of the GET band includes learners from Grades 7-9 (typically from 13-15 years of age).

2 A document camera is similar to an overhead projector, but it is more flexible and does not require the preparation of overhead slides. A document camera allows the facilitator/teacher/lecturer to project documents, notes, pictures and other objects on a screen or whiteboard.

3 The FET band/phase includes learners from Grade 10-12 (typically from $16-18$ years of age).

4 The Geometer's Sketchpad program is an interactive geometry software 
and the document camera.

The PowerPoint presentations for the lectures incorporated step by step methods of measuring, case studies of teachers teaching concepts within measurement and Euclidean Geometry, and various examples in the real world that required the knowledge and understanding of measurement (for example construction, engineering problems, driving and cooking/baking) and the use of geometry (art, video games, robotics, food design, graphic design, sports and architecture). The videos provided examples of how different teachers taught concepts in measurement and geometry within various contexts. The document camera was used to project notes and diagrams to engage students during lecture time so as to promote collaboration in order to support meaningful learning. Learning focused on concepts within measurement and geometry in mathematics. The Geometer's Sketchpad was used to illustrate to students how to interact dynamically with problems focusing on Euclidean geometry. Students were encouraged to use this dynamic program to construct and transform geometric shapes and functions in order to enhance learning.

Essentially students viewed the PowerPoint presentations and videos prior to the lecture so that they would have ideas and knowledge about the content that was going to be discussed during the lecture. During the lecture time the document camera and the Geometer's Sketchpad program was used to clarify and explain concepts further. At the end of the last lecture the participants were invited to one on one semi-structured interviews scheduled on dates a few months after the lectures. This meant that each participant would have the opportunity of reflecting on what they had learned from the lectures with the view of advancing their own pedagogy and thereby promoting their personal professional development. The interview was designed to gauge the perceptions of each participant on the use of the flipped learning approach within mathematics education in the era of the Fourth Industrial Revolution.

\subsection{The Lecture Observations}

Each lecture was observed using an observation schedule. The purpose of the observations was to ascertain if the flipped learning model was of benefit to the mathematics students. Hence, each lecture observation focused on student interaction during lecture time. The observations were also focused on whether or not the flipped learning environment encouraged student

program for exploring concepts within Euclidean geometry, algebra, calculus, and other sections in mathematics. You may download specific versions of Geometer Sketchpad program for free. This only allows you to preview Sketchpad sessions for a limited time period. This free preview does not allow you to print, save, copy or paste. A license needs to be purchased to allow for the effective use of the program in the classroom/lecture room. collaboration and discussion during the lecture. After completing each lecture observation, data from the observation schedule were inspected and segmented into meaningful codes. These codes were recorded for triangulation purposes.

\subsection{The Semi-structured Interviews}

The interviews were audio recorded and transcribed precisely. Interview transcriptions were sent to each of the thirteen participants for scrutiny to ensure the accuracy of each of the interview transcripts. The purpose of the interview was to ascertain the participants' perceptions of the flipped learning approach with the view of embracing the Fourth Industrial Revolution within Higher Education mathematics milieus. Each interview lasted approximately 35 minutes. The interviews were conducted at the participating university at a date and time that was convenient to each participant. Each interview began with a few general questions to place the participant at ease, the interview then progressed to specific questions focusing on the participant's perceptions of the use of the flipped learning approach. The findings of this study could provide valuable information for stakeholders in mathematics Higher Education within the ambit of embracing the Fourth Industrial Revolution.

\subsection{Data Analysis}

Data analysis which encompassed coding and categorizing of themes was based on the conceptual framework of the study, i.e. Activity Theory. The data analysis process focused on generating codes which described lecture observations and students' responses to the interview questions. Observations of each lecture was recorded using field notes and an observation schedule. Each interview was audio recorded and subsequently transcribed. All data generated were analyzed qualitatively, all transcribed interview data were read and reread line by line in-order for the researcher to become familiar with the data. After transcriptions of each interview were completed, the interview data were inspected and data were segmented into meaningful codes. The purpose of this type of coding was to reveal perceptions of participants focusing on the use of the flipped learning approach within the mathematics Higher Education milieu under study.

These codes were carefully reviewed by examining the relationship between the subject (student), the object (the teaching and learning of mathematics) and the tool (the flipped learning approach) within the activity system under focus. To triangulate the data analyzed, the interview codes were compared to codes that were recorded during the observations. The codes that were common from the interviews and observations were collated and captured as themes. For example, codes that reflected issues created by the resources were combined into the theme of material 
resources. Codes that spoke to aspects of the development or the training that was required by the lecturer were combined under the theme of lecturer professional development. Codes that were related to concepts being taught faster or time spent on discussion of concepts were collated under the theme of time spent in the lecture room. Lastly, codes that reflected aspects of collaboration and discussion were organized under the theme that focused on interaction and collaboration.

Thus, thematic coding was used to inductively create themes. These themes give a picture of how students perceived the use of the flipped learning approach for learning mathematics. The four main themes focusing on mathematics learning within a flipped learning environment that emerged from the qualitative data analysis of the observation schedules and interview transcripts are as follows. The flipped learning approach is inhibited by the lack of material resources, the flipped learning approach is dependent on lecturer professional development, the flipped learning approach saves time in the lecture room and the flipped learning approach encourages interaction and collaboration within the mathematics lecture room. These themes are discussed in detail in the section that follows.

\section{Results and Discussion}

While in general, the participants valued the flipped learning approach, they did indicate that they had misgivings and experienced problems whilst engaged within the flipped learning environment. This was also noted during the observations. Lecture observations and participants' responses to the interview questions are captured in the discussions that follow.

\subsection{The flipped Learning Approach is Inhibited by the Lack of Material Resources}

The students tried their best to download and view the videos and presentations prior to coming to the lecture, however some of the participants had challenges due to the lack of infrastructure or resources outside of the lecture room. These notions are supported by excerpts from the interview transcripts that follow.

Bongani:...it was fine...so excited to prepare for my lecture...we experienced load shedding 5 ...I could not access any presentations at home...get early to campus to

5 Load shedding refers to the interruption of an electricity supply to avoid excessive load on the generating plant. Load shedding is operational when there is an imbalance between the electricity available and the demand for electricity. These are planned interruptions in electricity supply by the Department of Electricity in South Africa. All consumers are informed in advance of load shedding times within their areas through the use of a comprehensive timetable. prepare from my lecture...

Brian: ...I could not access the video that was linked to the lecture...internet connection did not work properly at home...Telkom 6 was having problems...I was not prepared for the lecture...frustrating...

Kate:...it seemed like a good idea...combination of technology and the chalkboard...internet access is limited at home...stay after lectures to use the campus network...doesn't always work especially when my last lecture for the day was maths...

Simon:...I reflected on what I was exposed to during the lecture and I realized that while it would be beneficial...I do not have internet access or the appropriate gadgets at home...not willing to use by phone...too expensive...download presentations...my data for internet access is limited...

Based on the lecture observations, students spent the first few minutes of each lecture discussing the challenges they experienced regarding the out of lecture activities. This resulted in the lecturer playing video clips or presenting the PowerPoint presentations that were designed for out of lecture time during the first part of the lecture. Along similar lines, within the ambit of activity theory students ought to be supported by the lecturer however, the lack of material resources may hinder lecturers while they attempt to incorporate the flipped learning approach (FLA) within their lecture rooms. Thus, content and material needs to be intentional [16]. The preceding transcript excerpts indicate that students are willing to embrace the Fourth Industrial Revolution and they have a desire to embrace the FLA within their lecture rooms, however the lack of material resources affects their ability and confidence in technology enabled learning [24].

The effective integration of technology within teaching and learning is important in a $21^{\text {st }}$ century lecture room [25, 26] and technology is vital for embracing the Fourth Industrial Revolution (4IR). Lecturers ought to collaborate with their students to ensure that their individual contexts are adequately equipped with the basic resources required to embrace the flipped learning approach.

\subsection{The Flipped Learning Approach is Dependent on Lecturer Professional Development}

The effective integration of technology within the lecture room is an important part of lecturer professional development, lecturers need to know how to effectively use technology for instruction [27]. In this study, some students indicated that they were not satisfied with how the lecturer integrated technology-based tools within the flipped learning environment. They were of the notion that this integration was not effective and they frequently required more clarification. This perception is exemplified in the

6 Telkom is a telecommunications service provider in Africa. 
transcript excerpts that follow.

Busi:...I used the video to prepare for the lecture like you [the lecturer]7 told me to...it was OK then... when I came to the lecture...it was a bit different...the video just did not add up...it was not enough to answer and solve the problems...discuss the problems at length and then only it began to make sense...

Emma:...need help...how to use the technology tools...useful for learning...I need someone...show me how...work with the videos and PowerPoint...I think it would have helped...you [the lecturer] demonstrated in class first before giving us this to do...

Jane:...I just did not know how to link what was being discussed in the lecture with the PowerPoint I viewed at home... it was just too much of mixing with technology...it was an overload for me...I think that you [the lecturer] should have done this in stages and not as one whole presentation...I could not keep up...it was not a good feeling...left behind...

Veena:... I could not understand...couldn't explain how the video was related to the maths problems...I could only just do the first activity...it related to the formulation for the area of the building...I did not know how to solve the rest...it should have been clearer in your [the lecturers] video...the class discussed and assisted me...only then I got it...

Based on the lecture observations, the lecturer tried to assist students by exhibiting how to use the videos/technology-based tools on a step by step basis so as to clarify how to use the tool as well as to explain how the content of the videos/PowerPoint presentations were linked to the concepts under discussion. As is evident, there is a the need for the lecturer to be more aware of the students' needs within the flipped learning environment. Within the domains of the flipped learning model, there is a need to encompass the characteristics of a professional educator [13] whereby students are provided with clarification and feedback promptly. For example, the lecturer ought to acknowledge that all students learn differently and have different ability levels and this is important to consider within a technology-enabled environment. Thus, more professional development activities focusing on the effective use of information communication technology (ICT) for teaching within differing lecture room contexts and to students with differing ability levels are important [28]. So, while technology-based tools may be used to transform learning activities [29], lecturers require adequate professional development to become innovative in order to relate to their students and to effectively integrate ICT into teaching and learning within their lecture rooms [30].

7 Words in square brackets within the transcripts have been added by the researcher to support the reader's understanding.

\subsection{The Flipped Learning Approach Saves Time in the Lecture Room}

The lecturer transformed the lecture room while lecturing. Through a number of years of experience of teaching mathematics, the lecturer possessed the adequate content, pedagogic content and technological pedagogic content knowledge [31] to transform to a flipped learning environment successfully. The use of the technology-based tools assisted and empowered the lecturer to make this transformation. Based on the lecture observations and interviews, it was apparent that the participants valued the use of flipped learning approach and indicated that this approach also saved time during the lectures. This notion is evident in the transcript excerpts that follow.

Alec:...the videos supported me while I was preparing for the lecture...I could see the concepts...I could identify with what was being discussed...the videos saved time...time to have important discussions in class as well as more time to solve complicated problems...

Busi:...the PowerPoint presentation saved time when we were discussing the concepts in the lecture...go back to the slides to respond to queries and questions...since we already prepared for the lecture at home we only spent time discussing and improving our understanding...we also formed little groups to solve specific questions...we got together and solved...one person discussed the solutions to each problem in front of the entire class...we got much more done using the flipped method...

Rani:...for solving geometry...used Sketchpad8...we saved times since less time was spent on sketching the diagrams...we could refer back to the sketch to assist with questions and clarification and problem-solving...more accurate to draw using this technology teaching tool...we can visualize the transformations and manipulations...the sketches promoted detailed discussion...spent more time on clarification and discussion which helped with understanding...I was even thinking of changing the way I teach in school...

Sam:...we prepared for the lecture in advance so that in class we could refer to the complex problems and solutions as the class needed to...the diagrams became faster and easier to represent...they were already on the PowerPoint...the lecturer could spend more time guiding us and supporting us during the lecture...flipped method really works and more time is spent with discussing the important stuff...this can be easily replicated in my own classroom if I prepare adequately...

Based on the lecture observations, less lecture time was spent on introducing and clarifying concepts since the

8 Sketchpad is a type of dynamic geometry software that may be used to teach geometry in the lecture room. 
students had engaged with the content and concepts prior to the lecture. The lecture time was spent on problem-solving, collaboration and discussions. Based on the lecture observations, it was evident, that in this study, the flipped learning approach allowed the lecturer to teach content in innovative and interesting ways. The content taught was intentional and this maximized lecture time to encourage effective and meaningful learning $[15,16]$. The lecturer was an agent of change [32], the lecturer transformed the lecture room to embrace the 4IR and introduce the flipped learning approach to postgraduate mathematics students. The lecturer inspired the students by exhibiting innovative strategies for students to visualize the mathematics being taught. Being able to visualize the mathematical concepts being taught, inspires effective learning [33]. Moreover, within the ambit of activity theory, the learning environment under study was student centered and focused on engaging students in meaningful learning tasks [29]. Thus, technology-based tools within the flipped learning environment deepens students' understanding of concepts being taught and also enhances meaningful learning [34].

\subsection{The Flipped Learning Approach Encourages Interaction and Collaboration within the Mathematics Lecture Room}

Lecturers who aim to empower their students are prepared to hand over control of the learning process to their students, which ensures that learning becomes a collaborative effort rather than a coerced one [35]. This transformation in the learning process is different from the traditional learning process, the lecturer becomes a collaborator with the students.

Nomsa:... using the flipped method we use a lot of group work... it helps us... who do not understand English well... we come from different backgrounds and language groups...we work together ... to solve word problems...we access the presentations and videos before the lecture so we can prepare for the lectures...we talk to each other in the class to help with understanding concepts...its more interesting...it is not only working on your own...work together...

Collaborative relations of power within the lecture room empower students to achieve more. Collaboration and interaction within the lecture was evident from the observations and the interviews. The use of ... group work...helps the students who do not understand English well.... Students were encouraged to ... work together ... to solve word problems.... Students within these empowering education milieus know that their ideas are welcomed and respected. This was evident during all lecture observations, students spoke freely during lecture time and acknowledged the queries and feedback from both their peers and the lecturer. This learning culture ensured participation [13] within the lecture room and it was in this way that the lecturer inspired collaboration and interaction within this flipped education setting.

The participants believed that the use of the flipped learning approach (FLA) inspired collaborative learning within the activity system under focus. This was evident in all observed lectures. Hence, the students and lecturer used the FLA for collaborative mathematics learning. This learning culture supported and enhanced student learning [14]. The students could actively interact with each other and their lecturer and learn from these interactions. Moreover, within this study, participants indicated that collaborative learning through the use of the FLA as a tool, promoted mathematics learning. For some participants, collaborative learning supported by the FLA as a tool, shifted their tendency of individual mathematics learning to group learning within the activity system under focus. The interview excerpts that follow, support this notion.

Bongani:...I generally don't like asking questions in class...but I noticed that everyone was talking in class...I asked them to clarify a few questions...it was helpful...I did not feel embarrassed or self-conscious... I benefited from this interaction...I learnt a lot by working with my class and the lecturer...

Simon:... In all my lectures I generally work on my own...now for maths we work in a group...convenient for me...it helps with understanding difficult problems and topics...the flipped method allows me to prepare in advance and then in class we discuss issues that we have problems with...

Vernon:... the flipped method changed how I see my mathematics learning...I now enjoy collaborative learning since I can prepare in advance and then I can ask all the right questions...solve problems in class that I am struggling with...it is group work with a purpose...

In parallel with Bongani's, Simon's and Vernon's perceptions of the FLA as a tool, Veena acknowledged that collaborative mathematics learning took place within the activity system under focus by using the FLA as a tool. For Veena this demonstrated that she valued her peers' opinions and engaged with their explanations. She preferred this approach to her traditional self-study approach which entailed her solving mathematics problem on her own. This sentiment is evident in the interview excerpt that follows.

Veena: ... the flipped method made me realize how much my class are worth...inputs made by my class added to my mathematics learning...learnt a lot from my peers...

For Sam, he valued the fact that he and his peers could work collaboratively on a problem, but they still had the guidance and support of their lecturer. As soon as the students were confused or were found using an incorrect method to solve a problem, the lecturer would intervene and guide them to the correct solution. This is evident in the interview transcript that follows. 
Sam: ...some students have the same query...we help each other...since our lecturer is with us ... allows us to collaborate if we are ... on the wrong track...we are given guidance...this is useful...try the problems on our own and then we are provided with expert help [the lecturer] the moment we need it...

As is evident by the participants' response, each contribution made by students and the lecturer within the activity system through the use of the FLA as a tool led to positive outcomes for the student concerned. Similarly, Rambe and Bere [36] maintain that collaborative learning stimulates the construction of knowledge through group member interactions. Collaborative learning enhances the learning process, and motivates students to engage in academic activities which was the focus of the activity system under study. Collaborative learning also promotes the capabilities for cooperative work among students [37], this was evident in the observed lectures.

The value of collaborative learning is applicable to most disciplines and is not only confined to the teaching and learning of mathematics. Collaborative learning was particularly useful within this study and the use of the FLA served as an advantageous tool for the teaching and learning of mathematics within the activity system under focus. Moreover, through the use of the technology-based tools, students learn through participating in the process of contributing and receiving academic assistance, knowledge sharing and resolving challenges among themselves [38].

Also, due to the interactive and collaborative nature of the FLA, collaborative learning also improves throughput [18]. Generally based on the empirical evidence, there was consensus amongst the participants that the FLA as a tool for learning mathematics had provided them with the opportunity to learn mathematics collaboratively. Based on the observed evidence, the learning of mathematics through collaboration was welcomed and valued by the participants. The students within the activity system used the FLA as a tool to achieve the outcome of successful learning of mathematics. This tool was used to alleviate misconceptions and clarify concepts while students learnt mathematics. Through the flipped learning approach the students collaborated and discussed solutions whilst the lecturer facilitated. Collaboration is supported within the ambit of activity theory whereby, and student learning is enhanced by sharing and collaboration [39].

\section{Conclusions}

The aim of this study was to explore students' perceptions of using the flipped learning approach within a mathematics Higher Education lecture room. This qualitative, interpretive study was conducted at one university in KwaZulu-Natal, South Africa. Participants were observed while engaging within a flipped mathematics Higher Education lecture room. A sample of participants were subsequently interviewed. Four main themes emerged from a qualitative analysis of the interview data. The four themes are as follows: The flipped learning approach is inhibited by the lack of material resources, the flipped learning approach is dependent on lecturer professional development, the flipped learning approach saves time in the lecture room and the flipped learning approach encourages interaction and collaboration within the mathematics lecture room.

This study has provided some interesting observations and perceptions regarding the use of the flipped learning approach to embrace the Forth Industrial Revolution. It is evident that within contemporary lecture rooms, technology-based pedagogy has the potential to provide individualized, real time interactions among students and their lecturers [40]. This implies that lecturers may employ the use technology and social networks within their pedagogy in order to enhance meaningful learning within and outside their contemporary lecture rooms.

Moreover, based on the findings of this study it is apparent that within the contemporary lecture room, as technology becomes more available and if Activity Theory is embraced as an effective framework within the Fourth Industrial Revolution, lecturers will seek to engage in using notions of the flipped learning approach so as to amplify the benefits to student learning. This was evident since the lecturer within this study was willing to transform the traditional pedagogy to create a collaborative and engaging technology enriched lecture room so as to support students' learning needs. However, for the successful integration of the flipped learning approach within the contemporary lecture room, there is a need for lecturers to be involved in more professional development workshops focusing on how to enhance student learning while integrating the flipped learning approach within lecture rooms. These professional development workshops would of benefit to lecturers globally as we embrace the Fourth Industrial Revolution.

\section{Acknowledgements}

The author is grateful to the National Research Foundation (NRF) who partially funded this research study. NRF Grant Number: TTK170408226284, UID: 113952.

\section{REFERENCES}

[1] J.S. Pyper. Learning about ourselves: A review of the Mathematics Teacher in the Digital Era. Canadian Journal of Science, Mathematics and Technology Education. 2017;17(3):234-42.

[2] A. du Plessis. Editorial. Information and Communication Technologies and Educational Change. Educational Research for Social Change (ERSC). 2013;2(1):1-6. 
[3] L. Qing. Would we teach without technology? A professor's experience of teaching mathematics education incorporating the Internet. Educational Research. 2003;45(1):61-77.

[4] K. Schwab. The Fourth Industrial Revolution. Geneva, Switzerland.: World Economic Forum; 2016. p. 172.

[5] J. Butler-Adam. The Fourth Industrial Revolution and education. South African Journal Of Science. 2018;114(5):1.

[6] P. Rasanen, D. Laurillard, T. Käser, M. von Aster. Perspectives to technology-enhanced learning and teaching in mathematical learning difficulties. In: A. Fritz, V. Haase, P. Rasanen, editors. International handbook of mathematical learning difficulties. Germany: Springer; 2019.

[7] S. Pope, P. Mayorga. Enriching Mathematics in the Primary Curriculum. Exploring the Primary Curriculum. London: Sage; 2019.

[8] A.C.K. Cheung, R.E. Slavin. The effectiveness of educational technology applications for enhancing mathematics achievement in K-12 classrooms: A meta-analysis Best Evidence Encyclopedia [Internet]. 2011:[1-48 pp.].

[9] C.D. Bruce. Technology in the mathematics classroom: Harnessing the learning potential of interactive whiteboards What works? Research into Practice. 2012;38(1):1-4.

[10] H. Montrieux, R. Vanderlinde, T. Schellens, L. De Marez. Teaching and learning with mobile technology: A qualitative explorative study about the introduction of tablet devices in secondary education. PLOS ONE. 2015;10(12):1-11.

[11] G. Hwang, C. Lai, S. Wang. Seamless flipped learning: a mobile technology-enhanced flipped classroom with effective learning strategies. Journal of Computers in Education. 2015;2(4):449-73.

[12] R. Rajkumar, G. Hema. The flipped classroom: An approach to teaching and learning mathematics education. In: D. Chitra, U. Ramachandran, editors. International Conference on Teacher Education in Digital Era; IQAC, Kongunadu College of Education,. Tholurpatti, Thottiyam: Perambalur: Mercury Printers \& Publishers.; 2017. p. 753-7.

[13] N. Ramakrishnan, J.J. Priya. Effectiveness of flipped classroom in mathematics teaching. International Journal of Research-Granthaalayah. 2016;4(10):57-62.

[14] E.E. Demirel. Basics and key principles of flipped learning: Classes upside down. International Journal of Languages, Literature and Linguistics. 2016;2(3):109-112. doi:10.18178/ijlll.2016.2.3.77

[15] K. Sakulprasertsri. Flipped Learning Approach: Engaging 21st Century Learners in English Classrooms. LEARN Journal: Language Education and Acquisition Research Network Journal. 2017;10(2):132-143.

[16] S.M.P. Schmidt, D.L. Ralph. The flipped classroom: A twist on teaching. Contemporary Issues in Education Research. 2016; 9(1):1-6.

[17] N. Hamdan, P. McKnight, K. McKnight, K.M. Arfstrom. The flipped learning model: A white paper based on the literature review titled a review of flipped learning. Flipped Learning Network [Internet]. 2013:[1-16 pp.].

[18] C. Barhoumi. The effectiveness of WhatsApp mobile learning activities guided by activity theory on students knowledge management. Contemporary Educational Technology. 2015;6(3):221-38.

[19] D.H. Jonassen. Learning as activity. Educational technology. 2002;42(2):45-51.

[20] Y. Engeström. Learning by expanding: An activity-theoretical approach to developmental research. Helsinki: Orienta-Konsuit; 1987.

[21] S. Bødker, M.G. Petersen. Design for learning in use. Scandinavian Journal of Information Systems. 2000;12(1):61-80.

[22] L. Uden. Activity theory for designing mobile learning. International Journal of Mobile Learning and Organisation. 2007;1(1):81 - 102.

[23] E.W. Stein, V. Zwass. Actualizing organizational memory with information systems. Information Systems Research. 1995;6(2):85-117.

[24] E. Klopfer, S. Osterweil, J. Groff, J. Haas. Using the technology of today, in the classroom today. The instructional power of digital games social networking simulations and how teachers can leverage them. The Educational Arcade. 2006:1 - 23.

[25] D. Boothe, L. Clark. The 21st century classroom: Creating a culture of innovation in ICT2014. Available from: https://conference.pixel-online.net/ICT4LL/files/ict4ll/ed00 07/FP/0475-ICL733-FP-ICT4LL7.pdf.

[26] A.L. Cloete. Technology and education: Challenges and opportunities. HTS Teologiese Studies/Theological Studies. 2017;73(4):1-7.

[27] P.A. Ertmer, A. Ottenbreit-Leftwich. Removing obstacles to the pedagogical changes required by Jonassen's vision of authentic technology-enabled learning. Computers \& Education. 2012;64(1):175-82.

[28] R. Dlamini, K. Mbatha. The discourse on ICT teacher professional development needs: The case of a South African teachers' union. International Journal of Education and Development using Information and Communication Technology. 2018;14(2):17-37.

[29] R.N. Kizito. Connectivism in learning activity design: Implications for pedagogically-based technology adoption in African Higher Education contexts. International Review of Research in Open and Distributed Learning. 2016;17(2):19-39.

[30] D.E. Scott, S. Scott. Innovations in the use of technology and teacher professional development. In: J.O. Lindberg, A.D. Olofsson, editors. Online learning communities and teacher professional development: Methods for improved education delivery. Hershey, PA, USA: IGI Global; 2010. p. 169-89.

[31] P. Mishra, M.J. Koehler. Technological pedagogical content knowledge: A framework for teacher knowledge. Teachers College Record. 2006;106(6):1017 - 54.

[32] A.P. Ertmer, T.A. Ottenbreit-Leftwich. Teacher technology change: How knowledge, confidence, beliefs, and culture intersect. Journal of Research on Technology in Education. 2010;42(3):255 - 84.

[33] D.E. Shallcross, T.G. Harrison. Lectures: electronic presentations versus chalk and talk - a chemist's view. 
Chemistry Education Research and Practice. 2007;8(1):73 -

9.

[34] R. Huang, Y. Li. Examining the nature of effective teaching through master teachers' lesson evaluation in China. In: J. Cai, G. Kaiser, B. Perry, N-Y. Wong, editors. Effective mathematics teaching from teachers' perspectives National and cross-national studies. Rotterdam, The Netherlands: Sense Publishers; 2009. p. 163 - 81.

[35] T. Lawson. Empowerment in education: liberation, governance or a distraction? A review. Power and Education. 2011;3(2):89-103.

[36] P. Rambe, A. Bere. Using mobile instant messaging to leverage learner participation and transform pedagogy at a South African University of Technology. British Journal of Educational Technology. 2013;44(4):544-61.

[37] V.D. Tran. The effects of cooperative learning on the academic achievement and knowledge retention International Journal of Higher Education. 2014;3(2):131-40.

[38] N.M. Webb, A. Mastergeorge. Promoting effective helping behavior in peer-directed groups. International Journal of Educational Research. 2003;39(2):73-97.

[39] B. Duke, G. Harper, M. Johnson. Connectivism as a digital age learning theory. New York, USA: Kaplan University; 2013.

[40] P. Vululleh. Determinants of students' e-learning acceptance in developing countries: An approach based on Structural Equation Modeling (SEM). International Journal of Education and Development using ICT. 2018;14(1):141-51 